\begin{tabular}{l|l|} 
Postprint Version & 1.0 \\
\hline Journal website & $\underline{\text { http://www.jaacap.com }}$ \\
\hline Pubmed link & Http://www.ncbi.nlm.nih.gov/entrez/query.fcgi?cmd=Retrieve\&db=pubmed\&dopt=Abstr \\
\hline DOI & \begin{tabular}{l} 
act\&list_uids=16327586\&query_hl=15\&itool=pubmed_docsum \\
\hline $10.1097 / 01 . c h i .0000186402 .05465 . f 7$
\end{tabular} \\
\hline
\end{tabular}

\title{
Health Problems in Children and Adolescents Before and After a Man-made Disaster
}

ANJA J. E. DIRKZWAGER, PH.D., JAN J. KERSSENS, PH.D., AND C. JORIS YZERMANS, PH.D.

\begin{abstract}
Objective: The aims of this study were to examine health problems of children (4-12 years old at the time of the disaster) and adolescents (13-18 years old at the time of the disaster) before and after exposure to a fireworks disaster in the Netherlands (May 2000), to compare these health problems with a control group, and to identify risk factors for postdisaster psychological problems.
\end{abstract}

Method: Because the electronic medical records of family practitioners were used, longitudinal monitoring of health problems from 1 year predisaster until 2 years postdisaster for both victims $(\mathrm{N}=$ $1,628)$ and controls $(\mathrm{N}=2,856)$ was possible. Health problems were classified according to the International Classification of Primary Care.

Results: Postdisaster increases were significantly larger in victims than in controls for psychological problems, musculoskeletal problems, stress reactions, and symptoms of the extremities. Children 4-12 years old presented larger increases in sleep problems compared with controls, whereas children 1318 years old showed larger increases in anxiety problems than their controls. Significant predictors for postdisaster psychological problems included being relocated, presenting predisaster psychological problems, and a low to medium socioeconomic status.

Conclusions: Children and adolescents exposed to a disaster are at risk of long-lasting increases in both psychological and physical health problems. Postdisaster interventions should focus on those who were relocated and presented predisaster psychological problems. J. Am. Acad. Child Adolesc. Psychiatry, 2006;45(1):94-103.

On May 13, 2000, a fireworks depot exploded in a residential area in the city of Enschede in the Netherlands. As a result, a large part of the neighborhood was destroyed, leaving 500 uninhabitable houses and 1,000 damaged ones. This resulted in the necessary relocation of many residents (approximately 1,200). Twenty-two people, including four firefighters, were killed, and about 1,000 people were injured. After the disaster, a large-scale monitoring study was implemented to examine the health problems of the victims involved and the need for aftercare (Roorda et al., 2004). The present study focused on the health problems of the children involved in this disaster.

A growing body of literature demonstrates that both natural and man-made disasters are associated with a number of maladaptive reactions, such as posttraumatic stress disorder (PTSD), depression, anxiety, psychosomatic problems, and increased general distress (Donker et al., 2002; Green et al., 1994; Norris et al., 2002; North et al., 1999; Phifer, 1990). During the past decade, an increasing number of studies investigated the potential health effects among children who were victims of a disaster. Results showed that children exposed to disasters are also at risk of developing health problems afterward. For instance, posttraumatic stress symptoms, depression, anxiety, and behavioral problems such as enuresis or aggression have been found among children exposed to disasters (Bolton et al., 2000; Durkin et al., 1993; Goenjian et al., 1995; La Greca et al., 2002; March et al., 1997; Vogel and Vernberg, 1993). A recent systematic review of health consequences of disasters showed that compared with adult victims, child victims were more likely to experience severe impairments; 52\% 
of the schoolage samples experienced severe health effects after exposure to disasters compared with $42 \%$ of the adult samples (Norris et al., 2002).

The methodological quality of disaster research is often limited by the sudden, chaotic, emergency situation in which it has to take place. In the acute phase of a disaster, priority is given to the immediate trauma response or care for victims and not to concerns about the epidemiological study design. Therefore, longitudinal studies are rare, especially those comparing victims' health status before and after a disaster. The few studies on children that did include predisaster data found that predisaster anxiety, inattention, and academic skills were significantly associated with postdisaster PTSD symptoms (Asarnow et al., 1999; La Greca et al., 1998). The study of (Durkin et al. 1993) on the effects of a flood among 2 to 9 year olds demonstrated that the prevalence of aggressive behavior increased from 0 predisaster to nearly 10\% 5 months postdisaster, and one third of the children who had bladder control before the flood developed enuresis thereafter. Another study among very young children (23-44 months old) showed increased respiratory symptoms and coughs during the first 2 months following a hurricane (Simeon et al., 1993). In a recent study on mental health problems among adolescents who were exposed to a discotheque fire, both pre- and postdisaster data and data from controls were available; it was found that 5 months postdisaster adolescents exposed to the disaster had larger increases in self-reported anxiety and/or depressive problems, thought problems, aggression, and excessive alcohol use (Reijneveld et al., 2003).

The present study focused on the health problems of the children involved in the fireworks disaster, using the electronic medical records of family practitioners (FPs). In the Netherlands, every person is required to be registered at only one family practitioner, who must first be consulted if referral to secondary care is needed. Usually, each person has visited his or her FP at least once before a disaster strikes and has established a medical record. Medical records are a valuable source of information. Therefore, predisaster data on health are available. The aims of the present study were to examine the longitudinal course of health problems that children and adolescents who were exposed to a man-made disaster presented to their FP and to compare these health problems with those of a group of unexposed children. In addition, risk factors for postdisaster psychological problems were identified.

\section{METHOD}

\section{Participants}

All FPs $(N=60)$ in the city of Enschede were asked to participate in the study. Sixteen FPs (27\%) did not participate (mainly because of the expected increase in workload); $89 \%$ of all disaster victims appeared to be registered at the 44 participating FPs. To identify nonvictims (controls), a random sample was taken from the patients of the participating FPs who were not recorded as victims. This resulted in 20,055 patients who were registered with the participating FPs on the day of the disaster (9,329 victims and 10,726 controls). The percentage of patients who did not contact their FP during the study period ( 1 year predisaster to 2.5 years postdisaster) was small in both victims $(6.1 \%)$ and controls (7.7\%). Of the total patient group, 4,484 were children 18 years old or younger when the disaster occurred. Of these, 220 were disaster victims who had to be relocated and who lost all personal belongings because their houses were seriously damaged, 1,408 were victims who did not have to be relocated, and 2,856 were controls. The victims and controls did not differ significantly with respect to gender and age. Among the victims, slightly more than half were boys (54.1\%) and their mean age was 9.17 years $(\mathrm{SD}=5.62)$. Of the controls, $51.7 \%$ were boys and on average they were 9.14 years old $(\mathrm{SD}=5.46)$. The controls lived significantly more often in families with private health insurance (36\% for controls versus $32 \%$ for victims; $x^{2}=8.37$; $\mathrm{df}=1$; $\mathrm{p}<.01$ ).

Because we were interested in health consequences among both children and adolescents, we investigated two school-age samples: those who were 4-12 years old when the disaster occurred and those 13-18 years old at the time of the disaster. In the victim group, 752 children were 4-12 years old and 535 were 13-18 years old at the time of the disaster. For the controls, these numbers were 1,371 and 918, respectively.

\section{Measures}

The electronic medical records of the family practices were used. After each contact with a patient, the FPs register information on the presented health problems in their computer. All information on 
symptoms and diagnoses was used and classified according to the International Classification of Primary Care (ICPC), which is compatible with both the ICD-10 and the DSM-IV (Lamberts and Woods, 1987). Using individual ICPC codes will result in rather small numbers. Therefore, clusters of ICPC codes were composed (e.g., respiratory, gastrointestinal, musculoskeletal, psychological problems). The cluster of psychological problems consisted of ICPC codes representing stress reactions, anxiety problems, and depressive problems. Because previous disaster research suggested an increase in medically unexplained physical symptoms (MUPS) following disasters (Donker et al., 2002; Escobar et al., 1992), a cluster of MUPS was constructed containing symptoms, such as fatigue, abdominal pain, constipation, nausea, neck symptoms, backache, headache, shortness of breath, and coughing, from different organ systems.

In addition to the presented health problems, data on the following demographic characteristics were available: gender, age, and type of health insurance. The latter was used as an indication for socioeconomic status (SES) because in the Netherlands, people have private health insurance when their income is above a certain level (i.e., private health insurance represents a high SES, whereas a public health insurance represents a low to medium SES).

\section{Design and Procedures}

Because the existing registration systems of the FPs were used, longitudinal monitoring of health problems in the years before and after the disaster of both victims and controls was possible. In the present study, all of the data on health problems from 1 year predisaster until 2 years postdisaster were extracted from the electronic medical records in an anonymous format.

Disaster victims were identified in two different ways. First, they were marked by the FP, based upon the postal code of their home address at the time of the disaster or whether the disaster was discussed during consultation. Second, shortly after the disaster, an information and advice center (IAC) with a registration system of victims was implemented (everybody affected by the disaster could register at the IAC). Victims were included, either when the FP recorded them as a victim or when they were registered in the database of the IAC. A random sample of patients from the participating FPs who were not recorded as victims in either registration system (FP or IAC) and did not live in the disaster area constituted the control group.

Patients were informed about the participation of their FP in this monitoring study and could object to the use of their data. In 3 years, no one objected. In accordance with Dutch law, a detailed set of rules and regulations to protect the privacy of the respondents was followed, which had been approved by the Dutch Data Protection Authority.

\section{Data Analysis}

Prevalence rates of health problems per 1,000 people per year were calculated for the two age groups. Prevalence rates were calculated as the number of children presenting (one or more) symptoms divided by the number of children at risk, taking into account the amount of person-time during which events were counted as well as the time elapsed before health problems were presented (Rothman and Greenland, 1998).

We examined rates for the following clusters: psychological problems, medically unexplained physical symptoms, gastrointestinal problems, musculoskeletal problems, respiratory problems, skin problems, and social problems. These clusters included the most prevalent health problems, and together these clusters represent $52.6 \%$ of all problems presented to the FPs during the registration period. Because psychological and musculoskeletal problems appeared to be significantly increased in both age groups, we examined these clusters in more detail and calculated prevalence rates for a number of individual ICPC codes within these clusters (e.g., stress reactions, sleep problems, anxiety symptoms, back pain, neck symptoms).

Odds ratios with 95\% confidence intervals were used to compare victims with controls regarding changes between pre- and postdisaster rates. Logistic regression analysis was performed to examine factors associated with having presented psychological problems at the FPs' practices at least once during the first year postdisaster. The following independent variables were entered in the analysis: gender, insurance type, age, being a relocated victim (referenced to controls), being a nonrelocated victim (referenced to controls), and predisaster psychological problems. The interaction terms of the 
two victim groups (relocated and nonrelocated) with the other predictor variables were examined as well.

\section{RESULTS}

\section{Children 4-12 Years Old at the Time of the Disaster}

Before the disaster, victims and controls were similar in the number of psychological problems presented to the FP (Table 1). The prevalence rate of psychological problems of the 4- to 12-year-old victims increased dramatically in the first year postdisaster (from 23/1,000 predisaster to 209/1,000 postdisaster). In the second year, the prevalence of these problems decreased significantly but remained more than three times higher than before the disaster. The number of psychological problems among 4- to 12-year-old controls remained stable throughout time (about 20/1,000).

The odds ratios in Table 1 show the difference in change in health problems between victims and controls. A significantly larger increase in presented psychological problems postdisaster was found for victims when compared with controls. This increase was found for both the first- and second years postdisaster. A larger increase in victims than in controls was also found for musculoskeletal problems for both years postdisaster. In the first year postdisaster, significantly more victims presented social and gastrointestinal problems to the FP. The victims also had significantly higher odds ratios for MUPS, although this was mainly explained by a decrease of controls presenting such problems to their FP. A larger decrease was found in victims for respiratory problems postdisaster. Independent of the disaster, the victims showed higher prevalence rates for medically unexplained physical symptoms during all 3 years (i.e., pre- and postdisaster) when compared with controls.

With respect to individual ICPC codes, a significantly larger increase in stress reactions and sleep problems was found in victims compared with controls for both years postdisaster. During the first year postdisaster, larger increases were found in victims for learning problems and being overactive. For these two problems, the controls showed a decrease, whereas the victims showed an increase. The course of enuresis for this age group was also examined; no significant differences were found between victims and controls (not shown in Table 1). Long-term increases in physical problems were found as well. Two years postdisaster, victims reported larger increases in neck/back/shoulder/extremities symptoms than their controls.

\section{[ TABLE 1 ]}

\section{Children 13-18 Years Old at the Time of the Disaster}

Immediately after the disaster, a large increase was found in psychological problems among the 13to 18 -year-old victims (from 55/1,000 predisaster to $275 / 1,000$ postdisaster). Two years postdisaster, a twofold increase of such problems was found when compared with the predisaster level.

Table 2 shows the differences in change between the victims and controls 13 to 18 years old at the time of the disaster. Increases were significantly larger in victims than in controls for psychological problems, musculoskeletal problems, stress reactions, anxiety problems, shoulder symptoms, back pain, and symptoms of the extremities. Two years postdisaster, increases were still larger in victims for anxiety problems, back pain, and symptoms of the extremities. Two years postdisaster, victims also reported larger increases in skin problems than controls. Increases were significantly smaller in victims than in controls for medically unexplained physical symptoms, respiratory, and gastrointestinal problems.

Before the disaster, some differences between victims and controls already existed; victims presented more psychological problems, more MUPS, and more gastrointestinal problems than their controls.

\section{Comparison of the Two Age Groups}

In summary, a number of similarities were found between the two age groups. In both the 4 to 12 year olds and the 13 to 18 year olds, postdisaster increases were significantly larger in victims than in controls for psychological problems, musculoskeletal problems, stress reactions, and symptoms of the 
extremities. Some differences between the two age groups were also found. For instance, in the group who were 4 to 12 years old at the time of the disaster, the victims had signifi- cantly larger increases in gastrointestinal problems than their controls, whereas among those 13 to 18 years old when the disaster occurred, increases were significantly smaller in victims than in controls for these problems. Two years postdisaster, a significantly smaller increase in skin problems was found in the 4- to 12year-old victim group when compared with their controls.

Compared with their controls, a significantly larger increase in skin problems was found among the 13- to 18-yearold victims. Furthermore, the older group showed larger postdisaster increases in anxiety problems when compared with their controls, whereas the younger group presented larger increases in sleep problems compared with their controls.

\section{Risk Factors for Postdisaster Psychological Problems}

Because none of the interaction terms of the two victim groups (relocated and nonrelocated) with the other predictor variables were significant, Table 3 presents the results of the model without these interaction terms. Compared with controls, victims who were forced to relocate because of serious damage to their houses were especially at risk of presenting psychological problems to their FP in the first year postdisaster (odds ratio $=15.61$; Table 3 ). Children who were victims but did not have to relocate also reported significantly more psychological problems after the disaster compared with controls. In addition, children with psychological problems before the disaster, children of families with public health insurance, and older children were significantly at risk of postdisaster psychological problems.

\section{[ TABLE 2 ]}

\section{DISCUSSION}

The aims of the present study were to examine the health problems children and adolescents presented to their FPs before and after exposure to a man-made disaster and to compare these health problems with those of a control group. In addition, risk factors for postdisaster psychological problems were identified. It was found that psychological problems in particular were increased among the victims compared with both predisaster data and controls. During the first year postdisaster, the number of psychological problems increased by a factor of 9 among the younger victims (4-12 years old at the time of the disaster) and by a factor of 5 among the older victims (13-18 years old at the time of the disaster). Although the results of the individual health problems relate to rather small percentages, it was found that children who were older when the disaster happened presented larger increases in anxiety problems than their controls, whereas the children who experienced the disaster at a younger age presented more sleep and social problems compared with their controls. Although the prevalence rates declined with time, the number of psychological problems was still higher in victims than in controls 2 years postdisaster. These findings confirm the results of previous studies, showing that disasters can have long-term psychological consequences for children. In agreement with the present study, the controlled study of Reijneveld et al. (2003) found significant increases in anxiety and/or depressive problems among 12- to 15-year-old victims of a discotheque fire. The present study suggests that this increase may be related to increased anxiety problems rather than to increased depressive problems.

\section{[ TABLE 3 ]}

Increased physical symptoms, such as musculoskeletal problems, gastrointestinal problems (4-12 year olds), and skin problems (13-18 year olds) were also found among the victims. It cannot be ruled out that the increased physical symptoms may have been related to injuries received during the explosions. This is unlikely, however, because all injuries, such as burns, fractures, bruises, concussion, and internal injuries, that were externally caused are included in another cluster (injuries). Therefore, the clusters of physical symptoms used in this study did not include such injury-related problems. Because most previous research on the consequences of disasters for children focused on 
psychological or behavioral problems, little is known about potential physical health consequences. Studies on adult victims of disasters demonstrated physical problems after disasters. For instance, MUPS (Donker et al., 2002; Escobar et al., 1992), musculoskeletal problems (McFarlane et al., 1994; Schnurr et al., 2000), respiratory problems (McFarlane et al., 1994; Takakura et al., 1997), and increased gastrointestinal problems (Boscarino, 1997; Escobar et al., 1992) have been reported among adult victims of disasters. The present study on children also found increased musculoskeletal and gastrointestinal problems after the disaster, but respiratory problems were not increased among victims when compared with controls. Psychological problems and physical symptoms can be interrelated; studies have demonstrated that PTSD was related to cardiovascular, gastrointestinal, and musculoskeletal disorders (Hotopf et al., 1998; McFarlane et al., 1994; Schnurr et al., 2000). Thus, to examine the whole possible range of health consequences following disasters, future research on children involved in disasters should also pay attention to physical health consequences.

Before the disaster differences already existed in the prevalence of medically unexplained physical symptoms, with higher rates in the victim groups. Differences in socioeconomic status and ethnicity may have contributed to these predisaster differences because previous research suggested that these variables are associated with increased morbidity across different types of conditions. In the present study, slightly more victims came from families with a low to medium SES (i.e., public health insurance) compared with controls. Lack of information on ethnicity prevented an examination of this aspect.

Compared with controls, both victim groups (relocated and nonrelocated) presented significantly more psychological problems postdisaster. Those victims who lost their houses and had to be relocated for a long time in particular were at increased risk of postdisaster psychological problems. Relocation may be regarded as an indication of a higher degree of exposure because these children lived in the hardest-hit area, lost their homes and all of their personal belongings, and were more likely to experience traumatic situations such as seeing wounded or dead people. Furthermore, because they were relocated, they may have experienced a decrease in available social support. Previous studies suggested that forced relocation was a risk factor for psychological problems after the disaster (Lonigan, et al., 1994; Norris et al., 2002; Riad and Norris, 1996). Najarian et al. (1996, 2001) examined the effect of relocation after an earthquake disaster on both adults and children. Although adult women who were relocated reported significantly more depressive symptoms than nonrelocated women, no differences were found between relocated children and those who remained in the earthquake zone.

As noted previously, most studies did not have actual predisaster data; therefore, the available evidence of the effect of prior psychological functioning on postdisaster problems is scant. The present study fills this gap and shows that having presented predisaster psychological problems was associated with more psychological problems after the explosion. Two prospective studies on natural disasters showed similar results and found that predisaster anxiety was significantly associated with PTSD symptoms among children following a hurricane and an earthquake (Asarnow et al., 1999; La Greca et al., 1998).

In the present study, gender was not significantly related to postdisaster psychological problems, and a small but significant effect was found, indicating that older children were more likely to present psychological problems postdisaster. A number of previous studies indicated that girls reported more PTSD symptoms than boys following disasters, although the evidence regarding gender differences in youngsters is still mixed (La Greca et al., 2002; Norris et al., 2002). It is also likely that boys' and girls' postdisaster reactions depend on the type of health outcome that is studied; for instance, boys may be more likely to show aggressive behavior. Findings on age-related differences within young samples have been inconsistent as well (La Greca et al., 2002; Norris et al., 2002). For example, it was found that children younger than 8 years were less distressed after the Buffalo Creek dam disaster than children 8 to 15 years old (Green et al., 1991). The effects of Hurricane Hugo were found to be stronger for children 9 to 12 years old than among children 13 to 16 years old (Lonigan et al., 1994).

Public health insurance was significantly associated with more psychological problems postdisaster. This type of health insurance may be an indication of a low to medium SES because it is related to the annual income level. The role of socioeconomic characteristics in predicting youngsters' responses to disasters is not well investigated. Studies on adult populations consistently showed a positive relationship between low SES and postdisaster health problems (Norris et al., 2002). It has been 
argued that this may be related to the fact that people with a low SES possess fewer financial resources to deal efficiently with the recovery process.

\section{Limitations}

Before discussing the implications of the results, some methodological issues need to be considered. First, the study used the electronic registration systems of FPs. Although this approach reduces the problems associated with patient self-report (recall bias), it is possible that the FPs' interpretation of symptoms was influenced by the disaster, especially with patients marked as disaster victims. Second, to minimize interdoctor variation and to minimize regional differences between the exposed and nonexposed children, we used a control group of children from the same FPs. These controls were living nearby and may have been indirectly affected by the explosions. For instance, they may have heard the explosions or seen the disaster site in the days following the disaster or may have friends who were affected by the disaster. In this case, we may have underestimated the effects of the disaster, but we prefer the advantages over the disadvantages. Third, our study used the medical records as the source of information of health problems. Therefore, the study is limited to health problems children presented to their FP. This may reflect rather serious or enduring health problems. This different approach with physicians' diagnoses makes a direct comparison with studies based on self-reported data difficult but provides additional information on possibly the more objective health status of disaster victims. Finally, it is possible that the children's parents were more sensitized to health problems of their children after the disaster and more quickly took them to the FP. This may have resulted in an apparent increase in health problems. Even if this was the case, it is unlikely that this effect would last for 2 years, and this study still demonstrates the long-lasting impact of the disaster and the need for interventions.

The present study also has major strengths. Both comparisons with predisaster data and a control group were possible. Having such data is rather unique. Most studies on the health consequences of children following disasters are based on data from studies with only postdisaster cross-sectional data or only a control group comparison. Furthermore, most previous studies focused on psychological or behavioral problems among children following disasters. In the present study, the course of physical health problems was examined as well. Therefore, the present study adds valuable knowledge on the course of health problems among children and adolescents exposed to disasters.

\section{Clinical Implications}

What are the practical implications of these results? Children and adolescents exposed to a man-made disaster may suffer from long-term increased health problems. This warrants attention to this group of disaster victims and confirms the need for services aimed at children to try to prevent or decrease long-term negative health effects. Healthcare workers should be alert not only for psychological problems but also for physical health consequences among young disaster victims. The results emphasize the importance of postdisaster interventions aimed at stress reactions, anxiety problems, and sleep problems, but also at physical problems such as musculoskeletal or gastrointestinal problems. Some different health effects of the disaster were found within the two age groups, suggesting that interventions may need to take into account the age at which the child experienced the disaster. The ability to quickly identify high-risk children following a disaster is important for successful and effective prevention efforts. The results of the present study imply that children who lose their homes and personal belongings and have to be relocated who already had psychological problems before the disaster and who live in families with low to medium SES are particularly vulnerable and may benefit from an additional offer of help. 
Dirkzwager AJ, Kerssens JJ, Yzermans CJ.

Health problems in children and adolescents before and after a man-made disaster.

Journal of the American Academy of Child \& Adolescent Psychiatry, 2006 Jan;45(1):94-103

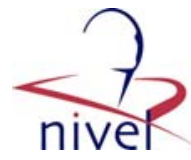

\section{TABLES}

TABLE 1

Number of 4- to 12 -Year-Old Children $/ 1,000 /$ Year Presenting Health Problems to the Family Practitioner, Grouped as Victims $(N=752)$ and Controls $(N=1,371)$

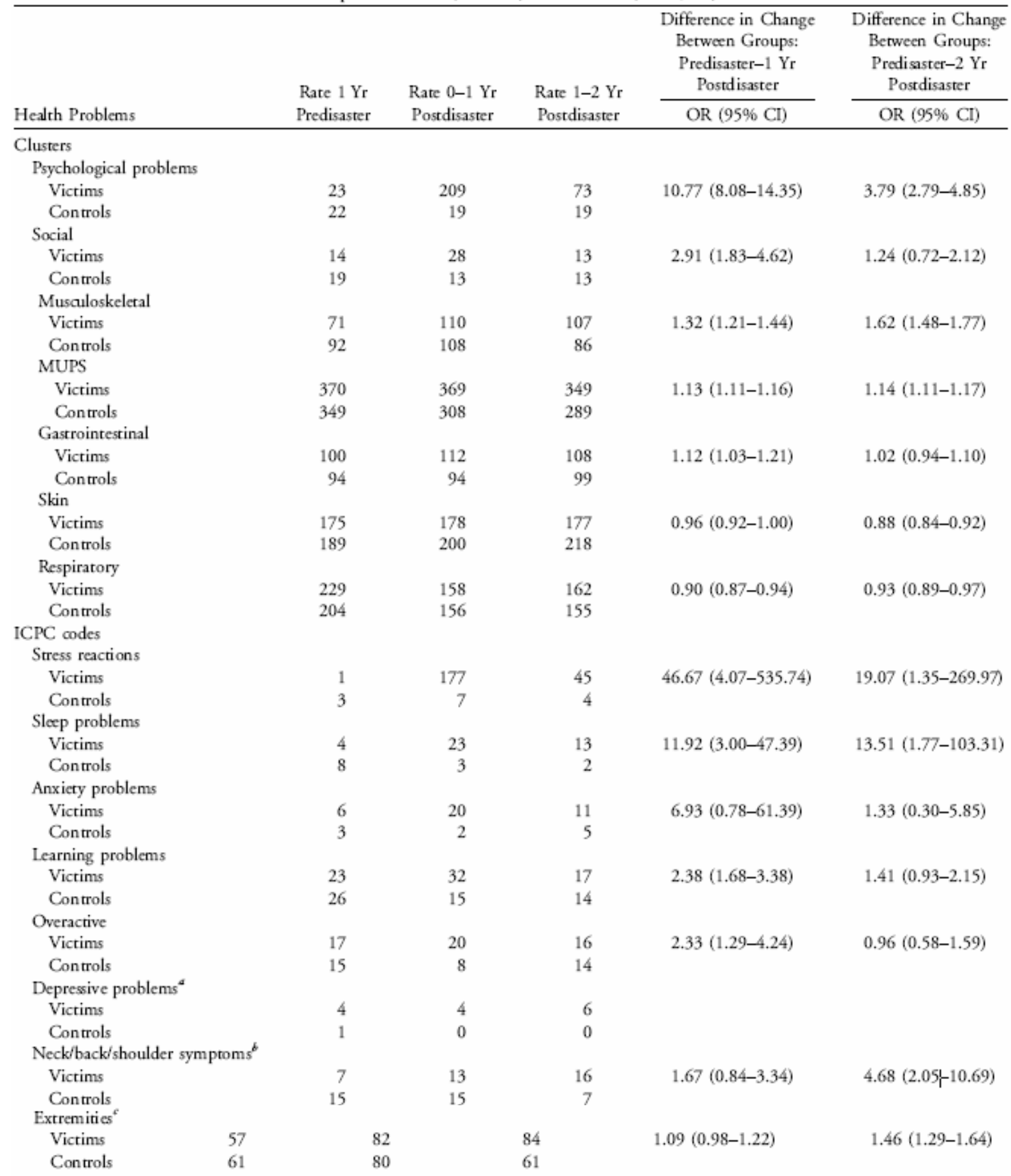


Dirkzwager AJ, Kerssens JJ, Yzermans CJ.

Health problems in children and adolescents before and after a man-made disaster.

Journal of the American Academy of Child \& Adolescent Psychiatry, 2006 Jan;45(1):94-103

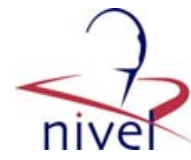

TABLE 2

Number of 13- to 18-Year-Old Children/1,000/Year Presenting Health Problems to the Family Practitioner, Grouped as Victims $(N=535)$ and Controls $(N=918)$

\begin{tabular}{|c|c|c|c|c|c|}
\hline Health Problems & $\begin{array}{l}\text { Rare } 1 \mathrm{Yr} \\
\text { Predisaster }\end{array}$ & $\begin{array}{l}\text { Rate 0-1 Yr } \\
\text { Postdisaster }\end{array}$ & $\begin{array}{l}\text { Rate } 1-2 \mathrm{Yr} \\
\text { Postdisaster }\end{array}$ & $\begin{array}{l}\text { Difference in Change } \\
\text { Between Groups: } \\
\text { Predisaster-1 Yr } \\
\text { Postdisaster } \\
\text { OR }(95 \% \mathrm{CI})\end{array}$ & $\begin{array}{c}\text { Difference in Change } \\
\text { Between Groups: } \\
\text { Predisaster-2 Yr } \\
\text { Postdisaster } \\
\text { OR }(95 \% \mathrm{CI})\end{array}$ \\
\hline \multicolumn{6}{|l|}{ Clusters } \\
\hline \multicolumn{6}{|c|}{ Psychological problems } \\
\hline Victims & 55 & 275 & 129 & $2.64(2.23-3.14)$ & $1.08(0.91-1.29)$ \\
\hline Controls & 24 & 44 & 50 & & \\
\hline \multicolumn{6}{|l|}{ Social } \\
\hline Victims & 35 & 76 & 51 & $1.27(0.94-1.72)$ & $0.57(0.42-0.76)$ \\
\hline Controls & 14 & 24 & 36 & & \\
\hline \multicolumn{6}{|l|}{ Musculoskeletal } \\
\hline Victims & 210 & 266 & 215 & $1.22(1.17-1.26)$ & $1.02(0.98-1.06)$ \\
\hline Controls & 198 & 206 & 198 & & \\
\hline \multicolumn{6}{|l|}{ MUPS } \\
\hline Victims & 457 & 515 & 502 & $0.96(0.94-0.97)$ & $0.87(0.85-0.88)$ \\
\hline Controls & 379 & 446 & 481 & & \\
\hline \multicolumn{6}{|l|}{ Skin } \\
\hline Victims & 200 & 198 & 251 & $0.96(0.92-0.99)$ & $1.37(1.32-1.42)$ \\
\hline Controls & 233 & 241 & 213 & & \\
\hline \multicolumn{6}{|l|}{ Respiratory } \\
\hline Victims & 159 & 161 & 178 & $0.91(0.86-0.96)$ & $1.02(0.97-1.07)$ \\
\hline & 130 & 145 & 143 & & \\
\hline \multicolumn{6}{|l|}{ Gastrointestinal } \\
\hline Victims & 101 & 99 & 100 & $0.63(0.57-0.70)$ & $0.54(0.49-0.60)$ \\
\hline Controls & 50 & 78 & 92 & & \\
\hline \multicolumn{6}{|l|}{ ICPC codes } \\
\hline \multicolumn{6}{|l|}{ Stress reactions } \\
\hline Victims & 6 & 232 & 66 & $9.67(2.69-34.82)$ & $3.72(0.95-14.64)$ \\
\hline Controls & 3 & 10 & 8 & & \\
\hline \multicolumn{6}{|l|}{ Anxiety problems } \\
\hline Victims & 14 & 24 & 36 & $2.57(1.35-4.88)$ & $2.14(1.30-3.53)$ \\
\hline & 12 & 8 & 14 & & \\
\hline \multicolumn{6}{|c|}{ Depressive problems } \\
\hline Victims & 8 & 20 & 24 & $0.83(0.21-3.22)$ & $0.87(0.24-3.21)$ \\
\hline Controls & 3 & 8 & 9 & & \\
\hline \multicolumn{6}{|l|}{ Sleep problems } \\
\hline Victims & 16 & 34 & 12 & $0.30(0.04-2.06)$ & $0.15(0.02-1.25)$ \\
\hline Controls & 1 & 9 & 6 & & \\
\hline \multicolumn{6}{|c|}{ Learning problems ${ }^{4}$} \\
\hline Victims & 4 & 8 & 4 & $0.99(0.05-20.27)$ & \\
\hline Controls & 1 & 3 & 0 & & \\
\hline \multicolumn{6}{|l|}{ Overactive $^{4}$} \\
\hline Victims & 0 & 0 & 1 & & \\
\hline Controls & 0 & 2 & 1 & & \\
\hline \multicolumn{6}{|c|}{ Shoulder symptoms } \\
\hline Victims & 12 & 24 & 14 & $2.24(1.23-4.09)$ & $0.81(0.45-1.56)$ \\
\hline $\begin{array}{l}\text { Controls } \\
\text { Back pain }\end{array}$ & 12 & 10 & 16 & & \\
\hline Victims & 35 & 61 & 61 & $1.44(1.21-1.72)$ & $1.22(1.03-1.45)$ \\
\hline Controls & 40 & 48 & 57 & & \\
\hline \multicolumn{6}{|l|}{ Extremities ${ }^{b}$} \\
\hline Victims & 124 & 162 & 125 & $1.36(1.28-1.45)$ & $1.27(1.19-1.37)$ \\
\hline Controls & 115 & 110 & 91 & & \\
\hline \multicolumn{6}{|l|}{ Neck symptoms } \\
\hline Victims & 27 & 24 & 43 & $0.85(0.58-1.24)$ & $1.33(0.96-1.85)$ \\
\hline Controls & 17 & 18 & 20 & & \\
\hline
\end{tabular}


Dirkzwager AJ, Kerssens JJ, Yzermans CJ.

Health problems in children and adolescents before and after a man-made disaster.

Journal of the American Academy of Child \& Adolescent Psychiatry, 2006 Jan;45(1):94-103

TABLE 3

Results of a Logistic Regression Analysis Examining Factors

Associated With Psychological Problems Presented to the FP During the First Year Postdisaster

\begin{tabular}{lrr}
\hline & OR & \multicolumn{1}{c}{$95 \%$ CI } \\
\hline Relocated victim & 15.61 & $10.13-24.05$ \\
Nonrelocated victim & 8.56 & $6.27-11.67$ \\
Psychological problems & & \\
$\quad$ predisaster & 5.72 & $3.41-9.62$ \\
Public health insurance & 1.61 & $1.21-2.14$ \\
Female gender & 1.26 & $0.99-1.62$ \\
Age, yr & 1.03 & $1.01-1.05$
\end{tabular}

\section{REFERENCES}

Asarnow J, Glynn S, Pynoos RS et al. (1999), When the earth stops shaking: earthquake sequelae among children diagnosed for pre-earthquake psychopathology. J Am Acad Child Adolesc Psychiatry 38:1016-1023

Bolton D, O'Ryan D, Udwin O, Boyle S, Yule W (2000), The long-term psychological effects of a disaster experienced in adolescence: II. General psychopathology. J Child Psychol Psychiatry 41:513-523

Boscarino JA (1997), Diseases among men 20 years after exposure to severe stress: implications for clinical research and medical care. Psychosom Med 59:605-614

Donker GA, Yzermans CJ, Spreeuwenberg P, van der Zee J (2002), Symptom attribution after a plane crash: comparison between self-reported symptoms and GP records. Br J Gen Pract 52:917-922

Durkin MS, Khan N, Davidson LL, Zaman SS, Stein ZA (1993), The effects of a natural disaster on child behavior: evidence for posttraumatic stress. Am J Public Health 83:1549-1553

Escobar JI, Canino G, Rubio-Stipec M, BravoM(1992), Somatic symptoms after a natural disaster: a prospective study. Am J Psychiatry 149:965-967

Goenjian AK, Pynoos RS, Steinberg AM et al. (1995), Psychiatric comorbidity in children after the 1988 earthquake in Armenia. J Am Acad Child Adolesc Psychiatry 34:1174-1184

Green BL, Grace MC, Vary MG, Kramer TL, Gleser GC, Leonard AC (1994), Children of disaster in the second decade: a 17-year follow-up of Buffalo Creek survivors. J Am Acad Child Adolesc Psychiatry 33:71-79

Green B, Korol M, GraceMet al. (1991), Children and disaster: age, gender, and parental effects on PTSD symptoms. J Am Acad Child Adolesc Psychiatry 30:945-951

Hotopf M, Mayou R, Wadsworth M, Wessely S (1998), Temporal relationships between physical symptoms and psychiatric disorder. Results from a national birth cohort. Br J Psychiatry 173:255261

La Greca AM, Silverman WK, Vernberg EM, Roberts ME (2002), Helping Children Cope With Disasters and Terrorism. Washington, DC: American Psychological Association

La Greca AM, Silverman WK, Wasserstein SB (1998), Children's predisaster functioning as a predictor of posttraumatic stress following Hurricane Andrew. J Consult Clin Psychol 66:883-892

Lamberts H, Woods M (1987), International Classification of Primary Care. Oxford, UK: Oxford University Press

Lonigan CJ, Shannon MP, Taylor CM, Finch AJ, Jr., Sallee FR (1994), Children exposed to disaster: II. Risk factors for the development of post-traumatic symptomatology. J Am Acad Child Adolesc Psychiatry 33:94-105

March JS, Amaya-Jackson L, Terry R, Costanzo P (1997), Posttraumatic symptomatology in children and adolescents after an industrial fire. J Am Acad Child Adolesc Psychiatry 36:1080-1088

McFarlane AC, Atchison M, Rafalowicz E, Papay P (1994), Physical symptoms in post-traumatic stress disorder. J Psychosom Res 38:715-726

Najarian LM, Goenjian AK, Pelcovitz D, Mandel F, Najarian B (1996), Relocation after a disaster: posttraumatic stress disorder in Armenia after the earthquake. J Am Acad Child Adolesc Psychiatry 35:374-383

Najarian LM, Goenjian AK, Pelcovitz D, Mandel F, Najarian B (2001), The effect of relocation after a natural disaster. J Trauma Stress 14:511- 526

Norris FH, Friedman MJ, Watson PJ, Byrne CM, Diaz E, Kaniasty K (2002), 60,000 disaster victims speak: Part I. An empirical review of the empirical literature, 1981-2001. Psychiatry 65:207-239 
Dirkzwager AJ, Kerssens JJ, Yzermans CJ.

Health problems in children and adolescents before and after a man-made disaster.

Journal of the American Academy of Child \& Adolescent Psychiatry, 2006 Jan;45(1):94-103

North CS, Nixon SJ, Shariat S et al. (1999), Psychiatric disorders among survivors of the Oklahoma City bombing. JAMA 282:755-762

Phifer JF (1990), Psychological distress and somatic symptoms after natural disaster: differential vulnerability among older adults. Psychol Aging 5:412-420

Reijneveld SA, Crone MR, Verhulst FC, Verloove-Vanhorick SP (2003), The effect of a severe disaster on the mental health of adolescents: a controlled study. Lancet 362:691-696

Riad JK, Norris FH (1996), The influence of relocation on the environmental, social, and psychological stress experienced by disaster victims. Environ Behav 28:163-182

Roorda J, van Stiphout WA, Huijsman-Rubingh R (2004), Post-disaster health effects: strategies for investigation and data-collection. Experiences from the Enschede firework disaster. J Epidemiol Community Health 58:982-987

Rothman KJ, Greenland S (1998), Modern Epidemiology. Philadelphia: Lippincott Williams \& Wilkins Schnurr PP, Spiro A, Paris AH (2000), Physician-diagnosed medical disorders in relation to PTSD symptoms in older military veterans. Health

Psychol 19:91-97 Simeon DT, Grantham-McGregor SM, Walker SP, Powell CA (1993), Effects of a hurricane on growth and morbidity in children from lowincome families in Kingston, Jamaica. Trans $R$ Soc Trop Med Hyg 87: 526-528

Takakura R, Himeno S, Kanayama Y et al. (1997), Follow-up after the Hanshin-Awaji earthquake: diverse influences on pneumonia, bronchial asthma, peptic ulcer and diabetes mellitus. Intern Med 36:87-91

Vogel JM, Vernberg EM (1993), Part 1: Children's psychological responses to disasters. J Clin Child Psychol 22:464-484 